\title{
ARTICLE
}

\section{Neuroanatomy of the fragile $X$ knockout mouse brain studied using in vivo high resolution magnetic resonance imaging}

\author{
R Frank Kooy ${ }^{1}$, Edwin Reyniers ${ }^{1}$, Marleen Verhoye $^{2}$, Jan Sijbers ${ }^{3}$, Cathy E Bakker ${ }^{4}$, \\ Ben A Oostra ${ }^{4}$, Patrick J Willems ${ }^{1}$ and Annemie Van Der Linden ${ }^{2}$ \\ Departments of ${ }^{1}$ Medical Genetics, ${ }^{2}$ Bio Imaging Lab, ${ }^{3}$ Physics, University of Antwerp, Antwerp, Belgium \\ ${ }^{4}$ Department of Clinical Genetics, Erasmus University, Rotterdam, The Netherlands
}

\begin{abstract}
Magnetic resonance imaging (MRI) of the brain of fragile $X$ patients, the most frequent form of inherited mental retardation, has revealed abnormalities in the size of specific brain structures, including the cerebellar vermis, the hippocampus, and the ventricular system. We intended to quantify the differences observed in the patient studies in the fragile $\mathbf{X}$ knockout mouse model, which is a good model for the disease, paralleling the human disorder in having cognitive deficits, macro-orchidism, and immature dendritic spines. Therefore we set up MRI of the mouse brain which allowed us to measure the size of the brain structures reported to be abnormal in human fragile $X$ patients in the mouse model. We did not find evidence for size alterations in various brain regions of the fragile $X$ mouse model, but the method described may find a wide application in the study of mutant mouse models with neurological involvement.
\end{abstract}

Keywords: MRI; fragile X syndrome; knockout mouse; mental retardation

\section{Introduction}

Fragile $\mathrm{X}$ syndrome is the most common cause of inherited mental retardation with an estimated frequency of $1 / 4000-6000{ }^{1}$ Besides moderate to severe mental retardation, features of the fragile $\mathrm{X}$ patient include macro-orchidism and specific dysmorphic features, as well as emotional and behavioural problems such as autistic features and hyperactivity. ${ }^{2}$ The disorder is caused by transcriptional silencing of the FMR1 gene on Xq27.3 by massive intragenic CGG repeat expansion. ${ }^{3,4}$ FMRP, the protein product of

Correspondence: Frank Kooy, Department of Medical Genetics, University of Antwerp, Universiteitsplein 1, 2610 Antwerp, Belgium. Tel: + 32 (0)3 8202630; Fax: + 32 (0)3 8202566; E-mail: fkooy@uia.ac.be

Received 24 September 1998; revised 12 March 1999; accepted 17 March 1999
$F M R 1$, is ubiquitously expressed, most abundantly in cerebral neurons and the Purkinje cells of the cerebellum. How the absence of FMRP causes the symptoms in the fragile $X$ patients is unknown, and the physiologic function of the FMRP is not well understood, although it is known that FMRP binds RNA.

Although cognitive dysfunction is the most relevant abnormality in fragile $\mathrm{X}$ syndrome, pathological studies of fragile $\mathrm{X}$ brains have been scarce. At a macroscopic level, no reproducible pathological abnormalities have been reported in eight separate adult post-mortem cases. $^{5-9}$ However, after microscopic examination, dendritic spine abnormalities were found in fragile $\mathrm{X}$ patients. Control persons show a mixture of stubby, mushroom shaped, and long, thin spines of the cortical pyramidal neurons after Golgi-staining. Pathological observations of the brains of three adult fragile $\mathrm{X}$ 
patients showed the presence of irregularly long and thin, tortuous spines mixed with very few normal short and stubby ones, whilst the number of neurons appeared within the normal range when compared with controls. ${ }^{7}$ Similar observations, though described in less detail, were made in a fragile $\mathrm{X}$ foetal brain sample. ${ }^{10} \mathrm{It}$ seems therefore that fragile $\mathrm{X}$ neurons possess synapses that resemble the immature synapses observed during neonatal development. It has been speculated that the immature synapses might be related to some aspects of the cognitive impairment in patients.

In contrast to neuropathological studies, abnormalities in the size of various brain structures of the fragile X patient were reported in studies using noninvasive methods, eg computed tomography (CT) scans or magnetic resonance imaging (MRI). CT scans of three patients revealed ventricular dilatation, exceeding the expected age alterations in one study. ${ }^{11}$ These findings were partially confirmed by a different study, demonstrating ventricular dilatation in eight patients out of 27. ${ }^{8}$ No evidence for ventricular dilatation or any other regional brain abnormality was found in the most recent study based on CT scan data comparing eight male fragile X patients with 20 healthy controls, but the right lateral ventricle appeared larger than the left in all but one of the patients. ${ }^{12}$

Most neuroanatomical data on fragile $\mathrm{X}$ brains is derived from MRI studies. MRI has a better resolution and tissue contrast than $\mathrm{CT}$ and in addition allows accurate quantification of the volumes of selected brain structures. Hypoplasia of the cerebellar vermis, the mid-sagittal plane of the cerebellum, was the first abnormality reported in a preliminary MRI study. ${ }^{13}$ The vermis is reported to be involved in sensory and motor integration, language, and modulation of agonistic behaviour. ${ }^{14}$ Thus, neuroanatomical vermis abnormalities might be related to some of the specific behavioural abnormalities observed in the fragile $\mathrm{X}$ syndrome, including hyperactivity, attention deficits, speech dysfluency, and perseveration. In addition, as a similar reduction in the size of the vermis has been observed in autistic disorders ${ }^{15}$ it was suggested that this abnormality might be related to the autistiform behaviour found in a subset of patients with the fragile $\mathrm{X}$ syndrome. Vermis hypoplasia was confirmed in subsequent MRI studies, comparing 14 fragile $\mathrm{X}$ males with 17 controls with a comparable level of developmental delay, but not fragile $\mathrm{X}$, and 18 controls with IQ values in the normal range (Table 1). ${ }^{14}$ The decrease in size was most prominent in the posterior vermis (lobules VI-X), notably in the lobules VI-VII, but not detected in the anterior vermis (lobules I-V). Volumetric data indicated no differences in cerebellar volume between fragile $\mathrm{X}$ males and controls, indicating the cerebellar abnormalities in patients are specifically restricted to the posterior vermis. Not unexpectedly, as fragile $\mathrm{X}$ female patients possess one functional copy of the FMRl gene, the decrease in posterior vermis area measured in 12 fragile $\mathrm{X}$ females was intermediate between male patients and a female control group, consisting of 12 IQ matched, non-fragile $\mathrm{X}$ controls. ${ }^{16}$ More recently, an inverse correlation between vermis area and the incidence of fragile $\mathrm{X}$-specific motor behaviour has been demonstrated. ${ }^{17}$ In addition, an association between the degree of vermis hypoplasia and cognitive performance was suggested. ${ }^{18}$ Along with the vermis hypoplasia, a $25-35 \%$ increase in size of the 4 th ventricle has been reported. ${ }^{13,14,16}$ It remains to be demonstrated whether this is a direct consequence of the size reduction of the vermis hypoplasia or of a more general increase of the ventricular system since the volume of the lateral ventricular cerebrospinal fluid was also increased by $60-80 \%$ in a group of 18 male patients. ${ }^{19}$

No size abnormalities were found in other posterior fossa or midline brain structures, including the corpus callosum, midbrain, pons, medulla, and third ventricle. ${ }^{14,16}$ However, when the volumes of temporal lobe structures of 15 young fragile $\mathrm{X}$ patients and 26 age and IQ matched controls were compared by MRI, an agerelated increase in both the left and right hippocampal volume, as well as an age-related decrease in the volume of the superior temporal gyrus was reported. ${ }^{20}$ Of temporal lobe structures, the hippocampus is potentially the most relevant to the pathogenesis of fragile $\mathrm{X}$ syndrome. It is a key structure in learning and memory, essential for the processing of visual-spatial information, a function that fragile $\mathrm{X}$ patients specifically perform worse than controls with a comparable level of developmental delay, but not fragile $\mathrm{X}$, in cognitive function tests. In contrast to the age-related decrease in hippocampal volume in younger patients, an MRI study by a different group of researchers found no evidence for a size alteration of the hippocampus in 16 adult patients. ${ }^{21}$

Fragile $\mathrm{X}$ patients also had a significantly larger volume of the caudate nucleus. ${ }^{19}$ This is a subcortical nucleus, which allows the cerebral cortex to affect behaviour, and thus this structure may be related to some of the behavioural abnormalities of fragile $\mathrm{X}$ 


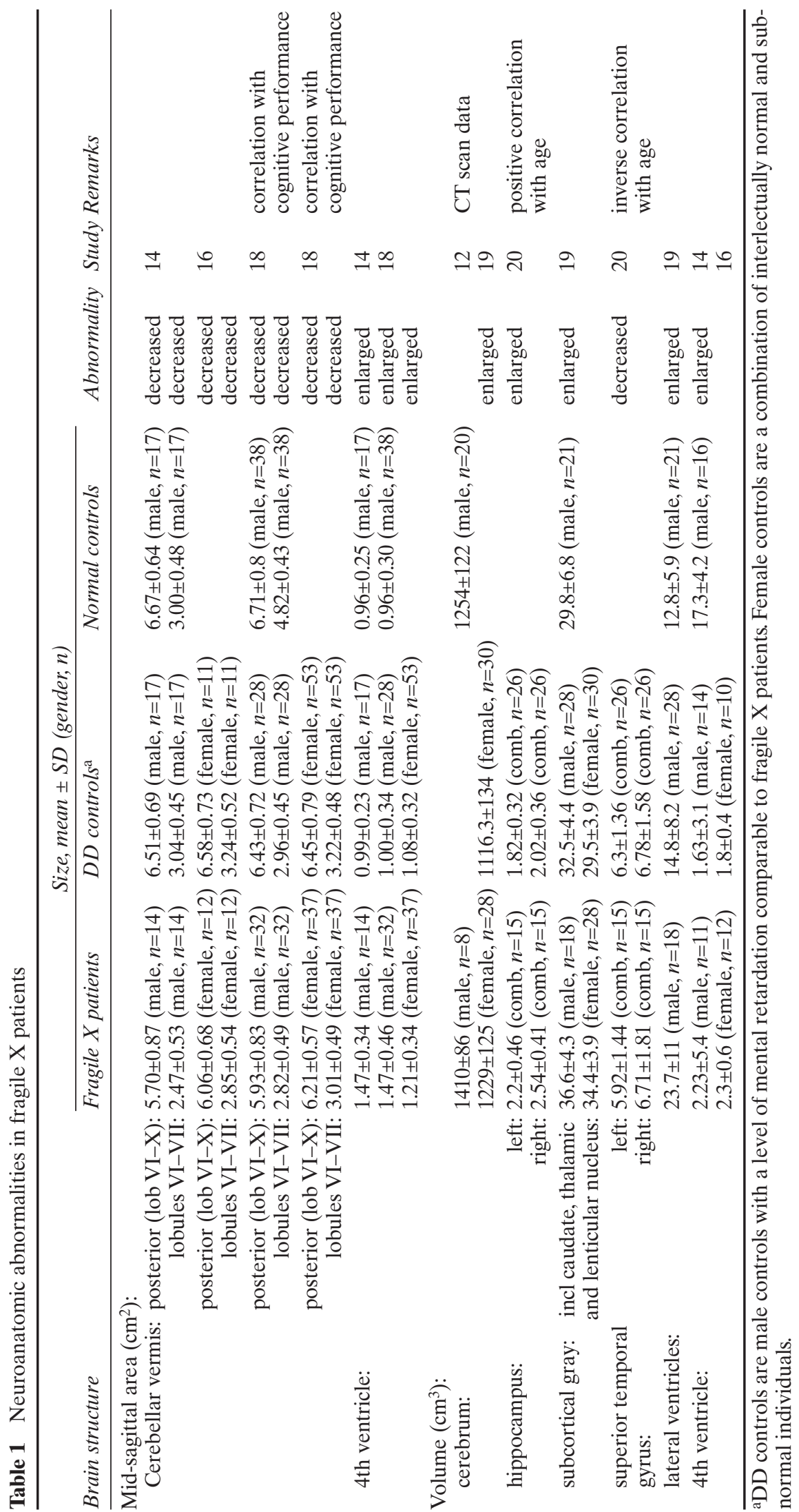


syndrome. Together with the lenticular nucleus (putamen), and the thalamic nucleus, the caudate nucleus forms the subcortical grey. In this same MRI study, an increase in the total brain volume in females but not in males was reported, whilst in an independent CT scan study a $12 \%$ increase in total brain volume in males was reported. ${ }^{12}$

Given the importance of the reported brain anomalies in understanding the pathogenesis of the fragile $\mathrm{X}$ syndrome and the function of the FMRl gene, we intended to quantify the brain anomalies in the fragile $\mathrm{X}$ knockout mouse. The fragile $\mathrm{X}$ knockout mouse produces no FMRP, and resembles human patients in showing impaired performance in cognitive function tests, macro-orchidism, and dendritic spine abnormalities. ${ }^{22-25}$ In parallel with studies performed on human patients, no other abnormalities were found in neuropathological studies of the knockout mouse brain. ${ }^{22}$ It therefore seems a true animal model for the human disorder. Using a mouse model will allow us to analyse the effect of the FMR1 mutation in a constant genetic background, an experimental set-up not possible with studies performed on human subjects.

\section{Materials and Methods}

\section{Animal Handling}

Eleven 70-day old male knockout and 11 70-day old male control FVB/N, 129 littermates were anaesthetised with a mixture of ketamine, xylazine and $0.9 \% \mathrm{NaCl}$ in a 2.1/0.3/3.4 mixture. Anaesthesia was induced by intramuscular injection of $0.1 \mathrm{ml}$ of this mixture, and maintained by subcutaneous injections of $0.05 \mathrm{ml}$ every half hour. This protocol proved sufficient to keep the mice motionless for prolonged periods of time and was non-lethal.

\section{MR Imaging}

In vivo MR imaging was performed at $300 \mathrm{MHz}$ on an SMIS MR system (SMIS, Guildford, UK) with a 7T horizontal bore magnet and $8 \mathrm{~cm}$ aperture self-shielded gradients with a strength of $100 \mathrm{mT} / \mathrm{m}$ (Oxford Instruments, Oxford, UK). By means of a stereotactical apparatus - ear plugs and a tooth bar - the head of the anaesthetised mouse was firmly fixed and positioned in the centre of a $30 \mathrm{~mm}$ wide RF bird cage coil used for both transmitting and receiving. Scouting spin echo images were first acquired to position the brain in the centre of the magnet and subsequently to orient the slab of the $3 \mathrm{D}$ acquisition.

To obtain high resolution sagittal images of the mouse brain within reasonable experimental time, a 3D Fast Spin Echo sequence ${ }^{26,27}$ was used with an echo train length of four, reducing the imaging time by a factor of 4 . MR signals of a 3D volume of $(20 \times 20 \times 22) \mathrm{mm}^{3}$ were acquired with a $(256 \times 128 \times 64)$ acquisition matrix. The images were taken with a repetition time of $2000 \mathrm{~ms}$ and an echo time of $25 \mathrm{~ms}$. The central line of k-space was sampled at the first echo.
These parameters were chosen so that the best compromise was made to obtain $3 \mathrm{D}$ images within an acceptable time, demonstrating a proper 4 th ventricle delineation together with a good contrast between grey and white brain tissue, permitting a segmentation of the cerebellum. Since optimum quality of the images was by sampling the central line of $\mathrm{k}$-space during the first echo, the use of longer TE (to obtain better $\mathrm{T}_{2}$-weighting) decreased the signal from later echoes, and thereby lowered the image quality. The imaging procedure took about $70 \mathrm{~min}$ after which all mice recovered. The raw MR data was zero-filled to $(256 \times 256 \times 256)$ for the image reconstruction. This zero-filling in the frequency space corresponds to sinc-interpolation in real space, and interpolation helps to determine the boundary of the structures to be segmented. This is because neighbouring pixels are strongly correlated (if not, the image would only contain white noise). It is very hard to quantify the increase in precision of the volume determination because that would require knowledge of the underlying (unknown) signal, but we believe that it can almost be doubled by doubling the data in the frequency space by zero-filling. Therefore, we assume an image matrix containing 256 sagittal slices of $86 \mu \mathrm{m}$ with spatial resolution of $(78 \times 78) \mu \mathrm{m}^{2}$

\section{Image Processing}

Image processing was performed on an HP 720 workstation. Brain structures were defined according to the mouse brain atlas. $^{28} \mathrm{~A}$ semi-automatic 3D segmentation technique ${ }^{29}$ was used to extract the brain and cerebellar volumes from 3D mouse MR images. Delineation of the 4th ventricle was based on intensity threshold selection, after reslicing the sagittal images to coronal images. For each data set, the threshold was consistently derived from the histogram of the total brain segmented data. The mid-sagittal areas of the entire brain, cerebellum and 4th ventricle were measured on the central slice applying the same segmentation procedure. Other areas were delineated by hand on expanded images: anterior and posterior cerebellum and subcortical grey on the mid-sagittal slice; and hippocampus on a sagittal slice at a distance of $0.6 \mathrm{~mm}$ from the central slice.

\section{Results}

The entire brains of 11 knockout and 11 controls were imaged; 256 sagittal slices were recorded from each animal, from one extreme end to the other of the mouse brain. The images were used to generate a 3D reconstruction of the entire mouse brain as described. ${ }^{29}$ Brain structures reported abnormal in human studies were identified with the aid of the atlas The Mouse Brain in Stereotaxic Coordinates. ${ }^{28}$

On the mid-sagittal section, total brain area of knockouts and controls did not differ significantly (Table 2). On the same section, the vermis of the cerebellum could be well delineated (Figure 1). Eleven knockouts showed an average vermis area of $12.7 \pm 0.8 \mathrm{~mm}^{2}$ (Table 2). This is identical to the controls, measuring $12.7 \pm 1.0 \mathrm{~mm}^{2}$. This is in line with 
Table 2 Brain volumes and surface areas in the fragile X knockout mouse

\begin{tabular}{|c|c|c|c|c|c|}
\hline \multirow[b]{2}{*}{ Total volume } & \multicolumn{2}{|c|}{ Knockout mouse } & \multicolumn{2}{|c|}{ Control mouse } & \multirow[b]{2}{*}{$P$} \\
\hline & $m e a n \pm S D$ & Range & $m e a n \pm S D$ & range & \\
\hline Total brain & $516 \pm 16$ & $492-540$ & $524 \pm 27$ & $484-570$ & ה19 \\
\hline Cerebellum & $70 \pm 3.1$ & $63.9-74.3$ & $74 \pm 7$ & $63.2-78$ & NS \\
\hline 4th Ventricle & $0.46 \pm 0.13$ & $0.33-0.69$ & $0.50 \pm 0.08$ & $0.35-0.62$ & NS \\
\hline \multicolumn{5}{|l|}{ Surface area } & \\
\hline Total brain & $76.0 \pm 2.4$ & $73.8-80.4$ & $76.5 \pm 3.2$ & $72.2-82.2$ & NS \\
\hline Cerebellum & $12.7 \pm 0.8$ & $11.3-14.0$ & $12.7 \pm 1$ & $11.5-14.2$ & NS \\
\hline Anterior (1-5) & $5.1 \pm 0.3$ & $4.6-5.6$ & $5.1 \pm 0.4$ & $4.3-5.6$ & NS \\
\hline Posterior (6-10) & $7.6 \pm 0.6$ & $6.6-8.6$ & $7.6 \pm 0.8$ & $6.3-9.0$ & \\
\hline 4th Ventricle & $1.7 \pm 0.2$ & $1.5-2.2$ & $1.8 \pm 0.3$ & $1.3-2.3$ & \\
\hline Subcortical gray & $4.1 \pm 0.2$ & $3.5-4.2$ & $4.0 \pm 0.1$ & $3.9-4.4$ & NS \\
\hline Hippocampus & $2.0 \pm 0.2$ & $1.8-2.3$ & $2.0 \pm 0.2$ & $1.7-2.3$ & \\
\hline
\end{tabular}

Values represent mean $\pm \mathrm{SD}$, volumes are expressed in $\mathrm{mm}^{3}$, surface areas in $\mathrm{mm}^{2}$. All surface areas are calculated from mid-sagittal slices, with the exception of the hippocampus, which was recorded on para-sagittal slices about $0.6 \mathrm{~mm}$ lateral from the mid-sagittal section. The mean of both para-sagittal hippocampal sections is provided. The anterior vermis was defined as lobules 1 to 5 and the posterior vermis as lobules 6 to 10. $P$ values were calculated using Student's $t$ test. NS, not significant.

human data, showing no difference in total vermis size between patients and controls (Table 1). However, in contrast to human data, no difference between posterior vermis size between knockouts and controls was measured. In line with this observation, as a reduction of the size of the cerebellar vermis results in an increase in the size of the 4 th ventricle, ${ }^{30}$ no significant difference in 4th ventricular area was observed between knockouts and controls.

The subcortical grey, consisting of caudate, lenticular, and thalamic nucleus, could also be delineated and measured at the mid-sagittal section, although it was not possible to identify each of the nuclei individually (Figure 1). In man, a significant difference between patients and controls in subcortical grey volume has been reported, but in the mouse model, the mid-sagittal area of knockouts $\left(4.1 \pm 0.2 \mathrm{~mm}^{2}\right)$ did not differ significantly from that of the controls $\left(4.0 \pm 0.1 \mathrm{~mm}^{2}\right)$. Although the hippocampus was discernible on the same section (Figure 1), measurements proved inconsistent, as the hippocampus is too small and not properly delineated on the mid-sagittal slice. Therefore, we measured the hippocampus on para-sagittal sections 7 slices (about $0.6 \mathrm{~mm}$ ) lateral from the mid-sagittal section - where the hippocampus is larger and well delineated from the surrounding brain structures. No significant difference between left and right section was measured and comparison of the mean hippocampal area from the knockouts $\left(2.0 \pm 0.2 \mathrm{~mm}^{2}\right)$ with the controls $\left(2.0 \pm 0.18 \mathrm{~mm}^{2}\right)$ revealed no difference between the two groups.

Using the 3D reconstruction of the mouse brain (Figure 2), it was possible to calculate the total brain volume and compare the knockout $\left(516 \pm 16 \mathrm{~mm}^{3}\right)$ with the control volume $\left(524 \pm 27 \mathrm{~mm}^{3}\right)$. In contrast to human fragile $\mathrm{X}$ brains which are larger than controls, no significant differences in size between knockout and control brains were found. The cerebellum was extracted from the cerebellum (Figure $2 \mathrm{~d}$ ) to allow size measurements of this structure separately from the rest of the brain, but no difference in size between control and knockout brains was registered. Using the same 3D reconstruction, it was possible to delineate the 4th ventricle on all slices, and thus determine its volume. No significant difference in size between knockouts $\left(1.7 \pm 0.2 \mathrm{~mm}^{2}\right)$ and controls $\left(1.8 \pm 0.3 \mathrm{~mm}^{2}\right)$ was found.

\section{Discussion}

We did not find evidence in the fragile $\mathrm{X}$ mouse model of size abnormalities of the brain regions reported to be abnormal in human fragile $\mathrm{X}$ patients, although we measured as many brain structures as possible which were reported abnormal in size in a series of human studies (see Table 1). Volumes of the cerebrum and the 4 th ventricle were calculated. It was not possible to measure the volume of the subcortical grey or the hippocampus in the mouse model, because the boundaries of these brain regions with the surrounding brain structures were not completely discernible on each section, so we used the mid-sagittal area instead as a measure of the size of these brain regions. In addition, the mid-sagittal area of the cerebellar vermis and the 4th ventricle, reported abnormal in at least five different human studies, were not different between knockouts and controls. 


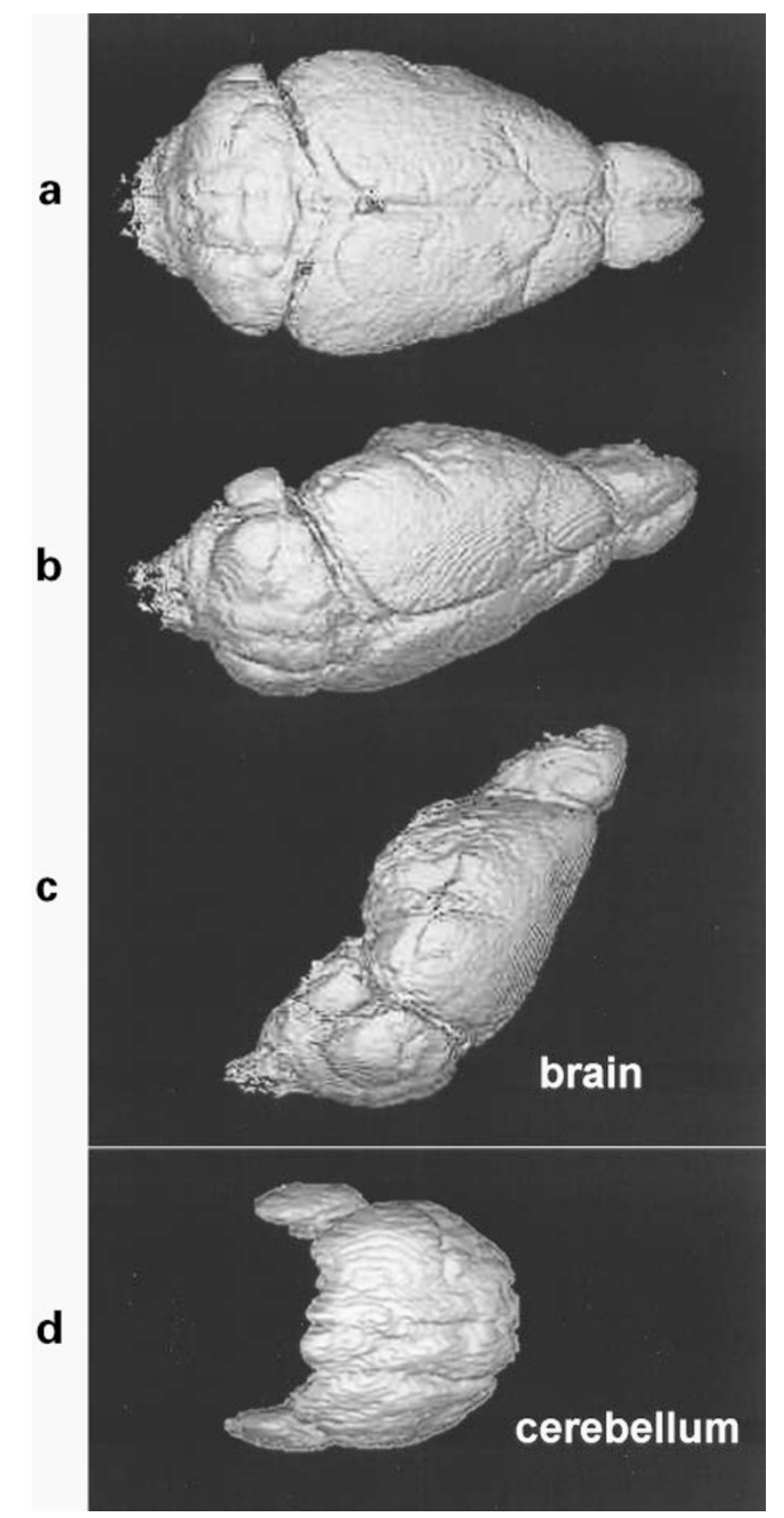

Figure 1 Different views of a 3-dimensional MRI reconstruction of the whole fragile $X$ mouse brain $(\mathbf{a}-\mathbf{c})$ and the cerebellum (d).

Two alternative explanations for the discrepancy between the human and murine pathology are possible. First the mouse model may simply not resemble the human disease with respect to brain structure, although the same neuropathologic abnormalities of dendritic spines have been reported in both. ${ }^{7,25}$ Alternatively, the structural differences in brain anatomy between human fragile $\mathrm{X}$ patients and controls may somehow have been overestimated in previous studies. Other preliminary human studies also point in that direction. ${ }^{21}$ In the latter respect, it is of interest to realise that the original

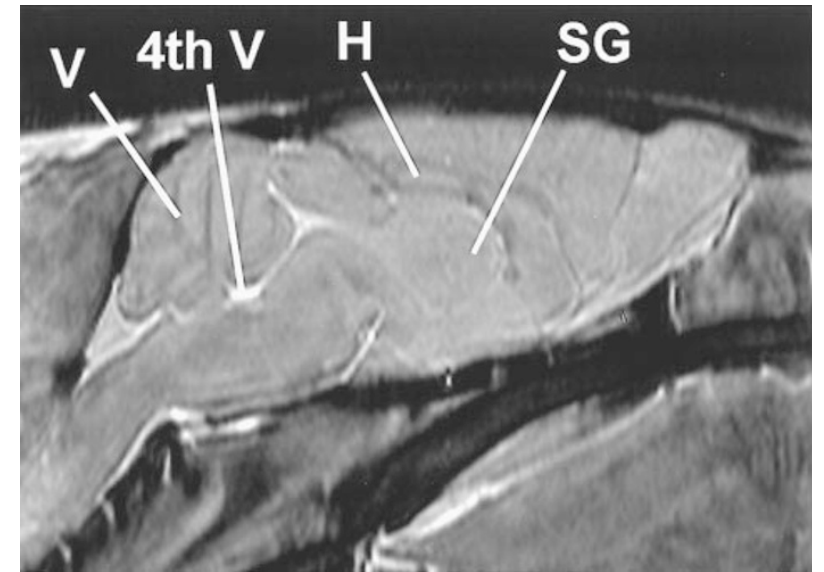

Figure 2 Mid-sagittal MR image from a fragile $X$ knockout mouse, illustrating the vermis (V), the 4th ventricle (4thV), the hippocampus $(H)$, and the subcortical grey $(S G)$.

finding that inspired the neuroanatomical studies on human fragile $\mathrm{X}$ patients, a correlation between autism and vermis size,, 15 was recently questioned. A size reduction of the posterior vermis was not exclusively found in a subset of patients with autism, but also in a percentage of patients suffering from neurogenetic syndromes without autistiform features. ${ }^{31}$

We studied here the neuroanatomy of a fragile $\mathrm{X}$ mouse model in living animals. The advantage of MRI recordings of living mice is that fixation artefacts are avoided and that the development of specific brain structures can be registered at several time points in the life of the same mouse. The images were of high resolution and allowed reliable and reproducible volume measurements of total brain, cerebellum, and 4th ventricle, and surface area determinations of several other brain regions. To our knowledge, in vivo high resolution MRI has not been used before to study brain anatomy from transgenic mouse models, ${ }^{32}$ and it can be expected that the protocol described here and in our parallel study on the L1CAM knockout mouse ${ }^{30}$ will be of wide use in the characterisation of mouse models of human disorders with neurological involvement.

\section{Acknowledgements}

We thank Dr Paul Parizel for help with the interpretation of the MRI images. Financial support for the fragile X syndrome research was obtained through grants from the University of Antwerp, the Belgian National Fund for Scientific Research, Flanders (FWO), the FRAXA research foundation, and from the EC. 


\section{References}

1 Kooy RF, Oostra BA, Willems PJ: The fragile X syndrome and other fragile site disorders. Results Probl Cell Differ 1998; 21: 1-46.

2 Hagerman RJ: Physical and behavioral phenotypes. In: Hagerman RJ, Cronister A (eds): Fragile Syndrome: Diagnosis, Treatment, and Research. Johns Hopkins University Press: Baltimore, MA, 1996, pp 3-87.

3 Verkerk AJMH, Pieretti M, Sutcliffe JS et al: Identification of a gene (FMR-1) containing a CGG repeat coincident with a breakpoint cluster region exhibiting length variation in fragile $\mathrm{X}$ syndrome. Cell 1991; 65: 905-914.

4 Pieretti M, Zhang F, Fu Y-H et al: Absence of expression of the FMR-1 gene in fragile X syndrome. Cell 1991; 66: $817-822$.

5 Dunn HG, Renpenning H, Gerrard JW, Miller JR, Tabata T, Federoff S: Mental retardation as a sex-linked defect. Am J Ment Defic 1963; 67: 827-848.

6 Desai HB, Donat J, Shokeir MHK, Munoz DG: Amyotrophic lateral sclerosis in a patient with fragile $\mathrm{X}$ syndrome. Neurology 1990; 40: 378-380.

7 Hinton VJ, Brown WT, Wisniewski K, Rudelli RD: Analysis of neocortex in three males with the fragile $\mathrm{X}$ syndrome. Am J Med Genet 1991; 41: 289-294.

8 Wisniewski KE, Segan SM, Miezejeski CM, Sersen EA, Rudelli RD: The Fra(X) syndrome: neurological, electrophysiological, and neuropathological abnormalities. Am J Med Genet 1991; 38: 476-480.

9 Reyniers E, Martin J-J, Cras P et al: Post-mortem examination of two fragile $\mathrm{X}$ brothers with an FMR1 full mutation. Am J Med Genet 1999; (in press).

10 Jenkins EC, Brown WT, Brooks J, Duncan CJ, Rudelli RD, Wisniewski HM: Experience with prenatal fragile $\mathrm{X}$ detection. Am J Med Genet 1984; 17: 215-239.

11 Veenema H, Geraedts JPM, Beverstock GC, Pearson PL: The fragile $\mathrm{X}$ syndrome in a large family. Cytogenetic and clinical investigations. Am J Med Genet 1987; 24: 23-31.

12 Schapiro MB, Murphy DGM, Hagerman RJ et al: Adult fragile X syndrome: neuropsychology, brain anatomy, and metabolism. Am J Med Genet 1995; 60: 480-493.

13 Reiss AL, Patel S, Kumar AJ, Freund L: Neuroanatomical variations of the posterior fossa in men with the fragile $\mathrm{X}$ (Martin-Bell) syndrome. Am J Med Genet 1988; 31: 407-414.

14 Reiss AL, Aylward E, Freund LS, Joshi PK, Bryan RN: Neuroanatomy of fragile $\mathrm{X}$ syndrome: the posterior fossa. Ann Neurol 1991; 29: 26-32.

15 Courchesne E, Yeung-Courchesne R, Press GA, Hesselink JR, Jernigan TL: Hypoplasia of cerebellar vermal lobules VI and VII in autism. $N$ Engl J Med 1988; 318: 1349-1354.

16 Reiss AL, Freund L, Tseng JE, Joshi PK: Neuroanatomy in fragile X females: the posterior fossa. Am J Hum Genet 1991; 49: 279-288.
17 Mazzocco MMM, Kates WR, Baumgardner TL, Freund LS, Reiss AL: Autistic behaviors among girls with fragile X syndrome. J Autism Dev Disord 1997; 27: 415-435.

18 Mostofsky S, Mazzocco MMM, Aakalu G, Warsofsky LS, Denckla MB, Reiss AL: Decreased cerebellar posterior vermis size in fragile $\mathrm{X}$ syndrome. Correlation with neurocognitive performance. Neurology 1998; 50: 121-130.

19 Reiss AL, Abrams MT, Greenlaw R, Freund L, Denckla MB: Neurodevelopmental effects of the FMR1 full mutation in humans. Nat Med 1995; 1: 159-167.

20 Reiss AL, Lee J, Freund L: Neuroanatomy of fragile X syndrome: the temporal lobe. Neurology 1994; 44: 1317-1324.

21 Jäkälä $\mathrm{P}$, Hänninen T, Ryynänen $\mathrm{M}$ et al: Fragile-X: neuropsychological test performance, CGG triplet repeat lengths, and hippocampal volumes. J Clin Invest 1997; 100: 331-338.

22 Bakker CE, Verheij C, Willemsen R et al: Fmr1 knockout mice: a model to study fragile $\mathrm{X}$ mental retardation. Cell 1994; 78: 23-33.

23 Kooy RF, D'Hooge R, Reyniers E et al: Transgenic mouse model for the fragile X syndrome. Am J Med Genet 1996; 64: $241-245$

24 D'Hooge R, Nagels G, Franck F et al: Mildly impaired water maze performance in male Fmrl knockout mice. Neuroscience 1997; 76: 367-376.

25 Comery TA, Harris JB, Willems PJ et al: Abnormal dendritic spines in fragile $\mathrm{X}$ knockout mice: maturation and pruning deficits. Proc Natl Acad Sci USA 1997; 94 5401-5404.

26 Yuan C, Schmiedl UP, Weinberger E, Krueck WR, Rand SD: Three-dimensional fast spin echo imaging: pulse sequence and on-vivo image evaluation. J Magn Reson Imaging 1993; 3: 894-899.

27 Zhou X, Cofer GP, Suddarth A, Johnson GA: High-field MR microscopy using fast spin echoes. Magn Reson Med 1993; 30: 60-67.

28 Franklin KBJ, Paxinos G: The Mouse Brain in Stereotaxic Coordinates. Academic Press: San Diego, CA, 1997.

29 Sijbers J, Scheunders P, Verhoye M, Van der Linden A, Van Dyck D, Raman E: Watershed-based segmentation of 3D MR data for volume quantization. Magn Reson Imaging 1997; 15: 679-688.

30 Fransen E, D’Hooge R, Van Camp G et al: L1 knockout mice show dilatated ventricles, vermis hypoplasia and impaired exploration patterns. Hum Mol Genet 1998; 7: 999-1009.

31 Schaefer GB, Thompson JN, Bodensteiner JB et al: Hypoplasia of the cerebellar vermis in neurogenetic syndromes. Ann Neurol 1996; 39: 382-385.

32 Martin JE, Fisher EMC: Phenotypic analysis - making the most of your mouse. Trends Genet 1997; 13: 254-256.

33 Kates WR, Abrams MT, Kaufmann WE, Breiter SN, Reiss AL: Reliability and validity of MRI measurement of the amygdala and hippocampus in children with fragile $X$ syndrome. Psychiatry Res 1997; 75: 31-48. 